\title{
El acceso público a la desfibrilación para los paros cardíacos mejoraría la sobrevida
}

\author{
Public-access defibrillation for out-hospital cardiac arrest may increase survival
}

\author{
Comentado de: \\ Kitamura $T$ y col. N Engl J Med 2016;375:1649-59. PMID: \\ $27783922^{1}$

\section{Objetivos}

Investigar los efectos de la diseminación de los programas de acceso público a la desfibrilación (APD) en la población de Japón sobre la sobrevida al mes y los resultados sobre el estado neurológico de los sobrevivientes, de las personas que sufren un paro cardiaco fuera del hospital.

\section{Diseño, lugar y participantes}

Estudio prospectivo, realizado en toda la población de Japón, desde 2005 a 2013. Se identificaron todos los paros cardiacos presenciados por fibrilación ventricular (FV) o de presunto origen cardíaco en los que se realizó un intento de reanimación.

\section{Desenlaces principales}

El estado neurológico se categorizó de acuerdo con la escala performance cerebral (de 1 a 5 ). Se estimaron los resultados de sobrevida y performance cerebral atribuidos al APD.

\section{Resultados}

Se identificaron 43.762 pacientes con FV o paro de presunto origen cardíaco, de los cuales 10,3\% recibieron APD. La primera observación para destacar del estudio fue el incremento temporal de los pacientes que recibieron APD, de $1,1 \%$ en 2005 a $16,5 \%$ en $2013(p<0,001)$. La sobrevida al mes y los resultados neurológicos favorables fueron mayores en aquellos que recibieron APD que los que no la recibieron ( $38,5 \%$ versus $18,2 \%$, con un odds ratio (OR) ajustado por un puntaje de propensión de 1,99 (Intervalo de Confianza [IC] del $95 \%: 1,80$ a 2,19). El número estimado de sobrevivientes al mes con un buen estado neurológico se incrementó por 6 veces desde 2005 a $2013(p<0,001)$.

\section{Conclusiones}

En Japón, el aumento del uso de la desfibrilación de acceso público por parte de los transeúntes se asoció con un aumento en el número de sobrevivientes con un resultado neurológico favorable después de un paro cardíaco por FV fuera del hospital.

\section{Comentario}

El manejo del paro cardíaco fuera del hospital ha cambiado definitivamente en las últimas décadas desde el advenimiento de los desfibriladores externos automáticos (DEA) a fines de la década de 1980, la posterior implementación de los programas de APD y el entrenamiento masivo del público general en reanimación cardiopulmonar. Estos programas fueron creados para optimizar el tiempo a la primera desfibrilación del DEA, en aquellos pacientes con paro cardíaco causado por una FV. Teniendo en cuenta que la tasa de sobrevida disminuye entre 10 y $15 \%$ por cada minuto en el que un paciente se halla en paro cardíaco por FV, y el desarrollo de daño neurológico se incrementa en igual proporción, es vital la implementación de programas de APD. El impacto de los programas de APD fue demostrado tempranamente, como en el trabajo de Capucci ${ }^{2}$, en la comunidad italiana de Piacenza donde en 2002 implementaron un programa APD triplicando la sobrevida de los paros cardíacos fuera del hospital solo con la implementación de DEA en lugares públicos a los que accedieron personas no entrenadas. Numerosos estudios posteriores confirmaron estos hallazgos en distintas poblaciones, como el estudio ROC (acrónimo del inglés Resuscitation Outcomes Consortium) ${ }^{3}$, en el que en una población de 21 millones de personas de 11 ciudades de USA y Canadá, la implementación de programas de APD duplicó la sobrevida de los paros cardíacos fuera del hospital entre 2005 y 2007. Estos hallazgos se fueron replicando en estudios realizados en diversas ciudades y países, y fueron acordes a los resultados de una revisión sistemática que comparó diferentes estrategias de APD: la desfibrilación por los primeros respondedores (personas sin entrenamiento profesional que presenciaran el evento) se asoció con la mayor supervivencia con una mediana de $53,0 \%$ (rango, 26,0 a 72,0), mientras que la realizada por profesionales con entrenamiento especial (bomberos, policía, etc.) fue de $28,6 \%$ (rango, 9,0 a 76,0$)^{4}$.

A nivel de sociedades científicas, desde mediados de la década de 1990, la Asociación Americana del Corazón recomienda la implementación de programas de APD ${ }^{5}$. El estudio comentado, confirma nuevamente la utilidad de los programas de APD, esta vez en una población no occidental.

\section{Conclusiones del comentador}

La realidad de nuestro país dista mucho de presentada en este trabajo. Existe una ley promulgada pero no reglamentada sobre el APD (Ley Nacional № 27.159). Numerosas instituciones privadas y públicas ya han implementado este tipo de programas, sin que existan estudios locales sobre su eficacia hasta la fecha.

Diego Iglesias [ Servicio de Cardiología, Hospital Italiano de Buenos Aires diego.iglesias@hospitalitaliano.org.ar ]

Iglesias D. El acceso público a la desfibrilación para los paros cardíacos mejoraría la sobrevida. Evid Actual Pract Ambul. 2019;22(3):e001073. Comentado de: Kitamura T, et al. Public-access defibrilation and out-of-hospital cardiac arrest in Japan. N Engl J Med 2016;375:1649-59. PMID: 27783922 


\section{Referencias}

1. Kitamura T, Kiyohara K, Sakai T, Matsuyama T, Hatakeyama T, Shimamoto T, et al. Public-access defibrillation and out-of-hospital cardiac arrest in Japan. New England Journal of Medicine. 2016;375(17):1649-1659. Available from: 10.1056/NEJMsa1600011.

2. Capucci A, Aschieri D, Piepoli MF, Bardy GH, Iconomu E, Arvedi M. Tripling survival from sudden cardiac arrest via early defibrillation without traditional education in cardiopulmonary resuscitation. Circulation. 2002 aug;106(9):1065-1070. Available from: 10.1161/01.CIR.0000028148.62305. 69.

3. Weisfeldt ML, Sitlani CM, Ornato JP, Rea T, Aufderheide TP, Davis D, et al. Survival after application of automatic external defibrillators before arrival of the emergency medical system: evaluation in the resuscitation outcomes consortium population of 21 million. Journal of the American College of Cardiology. 2010 apr;55(16):1713-20. Available from: 10.1016/j.jacc.2009.11.077.

4. Baekgaard JS, Viereck S, M $\varnothing$ ller TP, Ersb $\varnothing \| l$ AK, Lippert F, Folke F. The effects of public access defibrillation on survival after out-of-hospital cardiac arrest a systematic review of observational studies. Circulation. 2017;136:954-965. Available from: 10.1161/CIRCULATIONAHA.117.029067.

5. Aufderheide T, Hazinski MF, Nichol G, Steffens SS, Buroker A, McCune R, et al. Community lay rescuer automated external defibrillation programs: key state legislative components and implementation strategies: a summary of a decade of experience for healthcare providers, policymakers, legislators, employers, and community leaders from the American Heart Association Emergency Cardiovascular Care Committee, Council on Clinical Cardiology, and Office of State Advocacy. Circulation. 2006 mar;113(9):1260-70. Available from: 10.1161/CIRCULATIONAHA.106.172289. 